\title{
Synthetic TEC Mapping with Ordinary and Universal Kriging
}

\author{
I. Sayın ${ }^{1}$, F. Arıkan ${ }^{2}$, O. Arıkan ${ }^{3}$ \\ ${ }^{1}$ Hacettepe University, Department of Electrical and Electronics, isiltan@ee.hacettepe.edu.tr \\ 06800, Beytepe, Ankara, Turkey \\ ${ }^{2}$ Hacettepe University, Department of Electrical and Electronics, arikan@ $@$ hacettepe.edu.tr \\ 06800, Beytepe, Ankara, Turkey \\ ${ }^{3}$ Bilkent University, Department of Electrical and Electronics Engineering, oarikan@bilkent.edu.tr \\ 06800, Bilkent, Ankara, Turkey
}

\begin{abstract}
Spatiotemporal variations in the ionosphere affects the $\mathrm{HF}$ and satellite communications and navigation systems. Total Electron Content (TEC) is an important parameter since it can be used to analyze the spatial and temporal variability of the ionosphere. In this study, the performance of the two widely used Kriging algorithms, namely Ordinary Kriging (OrK) and Universal Kriging (UnK), is compared over the synthetic data set. In order to represent various ionospheric states, such as quiet and disturbed days, spatially correlated residual synthetic TEC data with different variances is generated and added to trend functions. Synthetic data sampled with various type of sampling patterns and for a wide range of sampling point numbers. It is observed that for small sampling numbers and with higher variability, OrK gives smaller errors. As the sample number increases, UnK errors decrease faster. For smaller variances in the synthetic surfaces, UnK gives better results. For increasing variance and decreasing range values, usually, the errors increase for both OrK and UnK.
\end{abstract}

\section{INTRODUCTION}

Ionosphere is a dispersive, temporally and spatially varying medium. This dispersive property of the ionosphere affects the performance of High Frequency (HF) and satellite communication and navigation systems. In HF communication, by using the reflection of electromagnetic waves from the ionospheric layers, communication through long distances can be made possible. The frequency at which the wave is reflected from the ionospheric layers is a function of electron density. Electron density depends on many factors such as solar activity, geomagnetism, latitude, local time and altitude. For the satellite systems, as the signals travel through the ionosphere, due to the changing refractive index, the signals are refracted and a delay error is observed in the received signal. For a better accuracy in the navigation systems and a continuous and qualitative communication, variability of the ionosphere have to be monitored continuously and corrections have to be made by using the gathered information.

Total Electron Content (TEC), which is defined as the number of free electrons in a cylinder of $1 \mathrm{~m}^{2}$ cross section, can be used to investigate the ionospheric variability. The unit of TEC is given in TECU where 1 TECU $=10^{16} \mathrm{el} / \mathrm{m}^{2}$. Ionosondes, incohorent backscatter radars, and satellite systems can be used for electron density or TEC computations. Global Positioning System (GPS) can be used to obtain TEC values with dual frequency receivers. With worldwide GPS satellites and receivers TEC computations can be made continuously. Due to sparse measurements in space and time accurate and robust estimation techniques are needed to better investigate the variability of the ionosphere. In this study, Ordinary Kriging (OrK) and Universal Kriging (UnK) algorithms, which are widely used methods in geostatistics are implemented on synthetic TEC surfaces, using the method applied in [1], with additional factors. Synthetic TEC surfaces are generated for a wide range of variance and correlation distances, with constant, linear, second order polynomial, Gaussian surface, and a more variable spatial trend surfaces. Synthetic TEC surfaces are sampled at a wide range of sample numbers and with various regular and random sampling patterns. For the regular patterns, square, triangular and hexagonal grids examined in [2] are used. For the random patterns uniform, inhibited, Poisson cluster point processes are generated as in [1]. For different sampling patterns, trend functions, variance and range values, performance of both OrK and UnK interpolation methods is compared. It is observed that for the regular sampling patterns, OrK and UnK give similar errors, but when the sampling is random, the method which assumes a wrong trend model has the largest errors. For the constant and the most variable trend, OrK has smaller errors, while for the other trends, generally, UnK has the smaller errors. For the increasing variance and decreasing range values, generally, error increases for all trends except the more variable trend funtion. For the constant trend, OrK gives smaller errors than UrK, but as the sample number increase, UrK errors get closer to OrK errors more rapidly than the OrK errors do for the scenarios in which UnK gives smaller errors than OrK.

In Section II, the random function model, which is a widely used model in many fields such as geology, geophysics, environmental sciences, is defined. OrK and UnK algorithms are given in Section III and implementation method and comparisons are given in Section IV and Section V. 


\section{RANDOM FUNCTION MODEL}

Due to the variations in the solar activity, geomagnetic storms, latitude, longitude and time, it necessary to have a statistical model. In environmental sciences, geology, hydrogeology, spatiotemporal models are used to investigate the behaviour of processes in nature which shows variability both in space and time. A finite domain in space and a finite domain in time can be defined as, $D \in R^{d}$ and $T \in R$, respectively. TEC can be modeled in space and time as a random function $\{Z(\mathbf{x}, t), \mathbf{x} \in D, t \in T\}$, where $Z(\mathbf{x}, t)$ is a random variable at $\mathbf{x}=\left[\begin{array}{ll}\theta & \phi\end{array}\right]^{\mathrm{T}}$, where $\theta$ latitude, $\phi$ longitude and at time $t$. For an instant of time, the region where TEC will be estimated, can be defined by a grid of $N_{\theta} N_{\phi}$ points, which has $N_{\theta}$ points in latitude and $N_{\phi}$ points in longitude. Points can be indexed by $1 \leq n_{\theta} \leq N_{\theta}$ in latitude and $1 \leq n_{\phi} \leq N_{\phi}$ in longitude. The lexicographical index $l=n_{\theta}+\left(n_{\phi}-1\right) N_{\theta}$ provides a mathematical ease to handle two dimensional matrix by resizing it into a one dimensional vector $\mathbf{g}_{s_{\left.U_{e} \mathbb{N}_{\varphi} \ngtr\right]}}$. Similarly, measurements at points $\mathbf{x}_{n_{a}}, n_{a}=1, \cdots, N_{a}$, can be defined by the vector $\mathbf{d}_{N_{a} \times 1}$. Mapping or interpolation can be considered as the problem of estimating the values in $\mathbf{g}_{s_{N_{e} N_{\phi} \times 1}}$ at grid points from the measurements $\mathbf{d}_{N_{a} \times 1}$. Estimations at the grid points can be given by the estimation vector:

$$
\hat{\mathbf{z}}_{s}=\left[\hat{Z}_{s}(1) \cdots \hat{Z}_{s}(l) \cdots \hat{Z}_{s}\left(N_{\theta} N_{\phi}\right)\right]_{1 \times N_{\theta} N \phi}^{T}
$$

where $\hat{Z}_{s}(l)$ is the estimation for the $l^{\text {th }}$ grid point. The random function $Z(\mathbf{x})$ is said to be strictly stationary if multivariate cumulative distribution function is invariant by translation $\mathbf{h}$. Since it is not possible to assure that this property is satisfied at all points, the statistical structure of the random function can be inferred from the set of point pairs $\mathbf{h}$ distance apart. In geostatistics, intrinsic stationarity, which is less demanding than the second order stationarity assumption, is employed for this purpose. An intrinsic stationary random function satisfies the below equations [6].

$$
\begin{aligned}
E\{Z(\mathbf{x})-Z(\mathbf{x}+\mathbf{h})\} & =m(\mathbf{h}) \\
\operatorname{var}\{Z(\mathbf{x})-Z(\mathbf{x}+\mathbf{h})\} & =2 \chi(\mathbf{h})
\end{aligned}
$$

In the above equations $E\{\}$ and $\operatorname{var}\{\}$ are the expectation and variance operators, respectively. $m(\cdot)$ is the drift function and $\gamma(\cdot)$ is the semivariogram function. In geostatistics, points that are close to each other are assumed to have similar values so generally the drift function is taken as zero [6]. When the second order stationarity is satisfied, semivariogram and covariance functions are related by the below equation.

$$
\gamma(\mathbf{h})=\operatorname{cov}(\mathbf{0})-\operatorname{cov}(\mathbf{h})
$$

In the above equation $\operatorname{cov}(\cdot)$ is the covariance function. When the stationarity assumptions are not satisfied the random function can be thought as the sum of a zero mean stationary random function $Y(\mathbf{x})$ and a trend $\mu(\mathbf{x})$ which is a function of coordinates [3].

$$
Z(\mathbf{x})=\mu(\mathbf{x})+Y(\mathbf{x})
$$

In the above equation $\mu(\mathbf{x})=E\{Z(\mathbf{x})\}$ can represent the trend in TEC values depends on space coordinates and $Y(\mathbf{x})$ can represent the variation above this trend function. Since distinct measurements related to $\mu(\mathbf{x})$ and $Y(\mathbf{x})$ are usually not available, physical information about the ionosphere can be used to estimate the structure of the trend functions.

\section{KRIGING INTERPOLATION METHOD}

Kriging is a widely used interpolation technique in geostatistics. It is first applied to mining, to estimate the ore grades in a mining block by D.G. Krige. Kriging linearly estimates the process by minimizing the error variance with respect to an unbiasedness condition. It also known as the Best Linear Unbiased Estimator (BLUE). A more detailed information about Kriging and geostatistics can be found in [4] to [6]. Geostatistics assumes that points close in space tend to have close values. So Kriging first preprocesses the data to infer the structure of variability of the random function. Experimental semivariogram which is the half of the variance of values at a constant distance apart is used for this purpose [4]. Calculation of experimental semivariogram from the data points is given as :

$$
\gamma^{*}(\mathbf{h})=\frac{1}{2 N(\mathbf{h})} \sum_{i \neq j}^{N(\mathbf{h})}\left[Z\left(\mathbf{x}_{i}\right)-Z\left(\mathbf{x}_{j}\right)\right]^{2}
$$

In the above equation $\mathbf{h}$ shows the distance lag between data points. $Z\left(\mathbf{x}_{i}\right)$ and $Z\left(\mathbf{x}_{j}\right)$ are the TEC values at points $\mathbf{x}_{i}$ and $\mathbf{x}_{j}$, respectively. $N(\mathbf{h})$ is the number of point pairs with a distance lag $\mathbf{h}$. Experimental semivariogram have to be fitted to a theoretical semivariogram function model $\gamma(\mathbf{h})$. Kriging estimate is the linear combination of values at measurement points. The estimation on the $l^{\text {th }}$ grid point defined in (1) is given in (7) for $N_{a}$ measurement points.

$$
\hat{Z}_{s}(l)=\sum_{n_{a}=1}^{N_{a}} w_{l ; n_{a}} Z\left(\mathbf{x}_{n_{\alpha}}\right), l=1 \cdots N_{\theta} N_{\phi}
$$

In (7), $w_{l ; n_{a}}$, for $1 \leq n_{a} \leq N_{a}$, are the Kriging coefficients for the $l^{\text {th }}$ grid point.

In Ordinary Kriging (OrK) a constant trend function is assumed. If the random function is intrinsic stationary the constant trend does not need to be known. For unbiased estimation the coefficients have to satisfy (8). 


$$
\sum_{n_{a}=1}^{N_{a}} w_{l ; n_{a}}=1
$$

Universal Kriging assumes a trend which is a linear combination of known functions with unknown coefficients as in (9).

$$
\mu(\mathbf{x})=E\{Z(\mathbf{x})\}=\sum_{n_{k}=1}^{N_{k}} a_{n_{k}} f_{n_{k}}(\mathbf{x}), \quad f_{1}(\mathbf{x})=1
$$

In (9), $a_{n_{k}}, 1 \leq n_{k} \leq N_{k}$, are the unknown trend coefficients, $f_{n_{k}}(\mathbf{x})$ are the known functions which are generally chosen as monomials to form a polynomial trend. For unbiased estimation, the coefficients have to satisfy (10).

$$
\sum_{n_{a}=1}^{N_{a}} w_{l ; n_{a}} f_{n_{k}}\left(x_{n_{a}}\right)=f_{n_{k}}\left(x_{l}\right), \text { for } n_{k}=1, \ldots, N_{k}
$$

Estimation variance for the $l^{\text {th }}$ grid point can be given by (11).

$$
\sigma_{l}^{2}=2 \sum_{n_{a}=1}^{N_{a}} w_{l ; n_{a}} \gamma\left(x_{n_{a}}, x_{l}\right)-\sum_{n_{a}=1}^{N_{a}} \sum_{n_{b}=1}^{N_{b}} w_{l ; n_{a}} w_{l ; n_{b}} \gamma\left(x_{n_{a}}, x_{n_{b}}\right)
$$

For both OrK and UnK, coefficients can be estimated with the Lagrange multiplier method by minimizing the estimation error variance while satisfying the unbiasedness constraints [4], [5]. The needed semivariogram values between the points can be calculated from the fitted theoretical semivariogram function. The spatial interpolation performances of OrK and UnK algorithms will be compared on the synthetic TEC surfaces in the next section.

\section{SyNTHETIC TEC INTERPOLATION}

Since a complete forward model of the ionosphere does not exist and since the measurements both in space and time are sparse, it is necessary to test the performance of the interpolation techniques first with synthetic surfaces. In this section, a comparison of the performance of OrK and UnK algorithms on synthetic TEC surfaces is given by using a similar method followed by [1] and for an additional sample number factor and for various trend types. A grid, defined in Section II, is chosen on the midlatitude region, for $N_{\theta}=11$, $N_{\phi}=24$ corresponding to $N_{\theta} N_{\phi}=264$ points in total, with $1^{0}$ resolution both in latitude and longitude. For various ionospheric states such as quiet and disturbed days, trend function in (5) can be chosen as :

$$
\begin{aligned}
& \mu_{1}(\mathbf{x})=a_{11} \\
& \mu_{2}(\mathbf{x})=a_{12}+a_{22} \theta+a_{32} \phi \\
& \mu_{3}(\mathbf{x})=a_{13}+a_{23} \theta+a_{33} \phi+a_{43} \theta^{2}+a_{53} \theta \phi+a_{63} \phi^{2}
\end{aligned}
$$

$$
\mu_{4}(\mathbf{x})=a_{14}+\exp \left(-\left(\frac{\theta-\theta_{04}}{a_{24}}\right)^{2}-\left(\frac{\phi-\phi_{04}}{a_{34}}\right)^{2}\right)
$$

$$
\mu_{5}(\mathbf{x})=a_{15}+\exp \left(-\left(\frac{\theta-\theta_{05}}{a_{25}}\right)^{2}-\left(\frac{\phi-\phi_{05}}{a_{35}}\right)^{2}\right)
$$

$\mu_{6}(\mathbf{x})=\sqrt{a_{16} \cos ^{2} \theta+a_{26} \sin ^{2} \phi}-\exp \left(a_{36}(\cos \theta+\cos \phi)\right)$

The coefficients of the trend functions given by (12) to (15) are chosen such that the functions represent the TEC values for a quiet day of the ionosphere for different time instants for a day and to represent a trend from north to south. Trend functions (16) and (17) represent a disturbance in the grid of interest. The center of the Gaussian function in (16) is in the middle of the grid, while that of (15) is below the south of the grid. Minimum and maximum of the TEC values are 15 and 25 TECU, respectively, in all trend functions.

Cholesky Decomposition, which is a geostatistical data simulation technique [4], is used to simulate a zero mean, Gaussian, spatially correlated random function $Y(\mathbf{x})$ as in (5). The variance and range of correlation is determined by an exponential covariance function in (18).

$$
\operatorname{cov}(\mathbf{h})=\sigma^{2} \exp \left(\frac{-|\mathbf{h}|}{a}\right)
$$

For the variance $\sigma^{2}$ of the residual random function $Y(\mathbf{x})$, one of the $0.64,1.44$, and 2.56 values is chosen for different variability levels. For a wide range of correlation distances the values 5,10 or 15 are chosen for the range $a$ of the residual random function.

Sampling points are located regularly as square, triangular and hexagonal grids [2], and randomly as uniform, inhibited and clustered [1], for different sampling numbers $20(7.6 \%), 30$ $(11.4 \%), 40(15.2 \%), 50(19.0 \%), 60(22.7 \%)$ and 70 (26.5\%). In each scenario, residual synthetic TEC data $Y(\mathbf{x})$, is generated at the grid points and the sampling points, for each option of the sampling pattern, sample number, variance $\sigma^{2}$ and range $a$. Then $Y(\mathbf{x})$ is added to the one of the possible trend functions $\mu(\mathbf{x}) .10$ realizations of each scenario are generated. Kriging methods OrK and UnK with a second order polynomial trend are used for estimating the synthetic TEC values at grid points $\mathbf{g}_{s}$ from the values at sampling points $\mathbf{d}$ and generating the estimation vector $\hat{\mathbf{z}}_{s}$ given in (1). For both of OrK and UnK, the semivariogram function is calculated by using a known covariance function, as in (18). For each realization of a scenario, the normalized error is given by

$$
\varepsilon_{n}=\frac{\left\|\hat{\mathbf{z}}_{s}-\mathbf{g}_{s}\right\|^{2}}{\left\|\mathbf{g}_{s}\right\|^{2}}
$$


The average normalized error $\left(\varepsilon_{n}\right)_{a v}$ for one scenario is the average of the normalized errors for all realizations of the scenario. The performance of UnK with respect to OrK is evaluated with a relative error $\mathcal{E}_{r}$ criteria :

$$
\varepsilon_{r}=\frac{\left(\varepsilon_{n}\right)_{a v}^{U n K}-\left(\varepsilon_{n}\right)_{a v}^{O r K}}{\left(\varepsilon_{n}\right)_{a v}^{O r K}} \times 100
$$

When UnK gives smaller errors, $\mathcal{E}_{r}$ becomes negative, when UnK gives larger errors $\varepsilon_{r}$ becomes positive. Table I. shows the typical maximum $\varepsilon_{r}$ values for all trends when the sample number increases, for a regular square sampling pattern and a random uniform sampling pattern.

TABLE I

Typical maximum relative errors as the sample number increases

\begin{tabular}{|c|l|l|}
\hline & \multicolumn{2}{|c|}{ Sampling Pattern } \\
\hline Trend Function & \multicolumn{1}{|c|}{ Regular (Square) } & Random(Uniform) \\
\hline$\mu_{1}(\mathbf{x})$ & $5 \%$ to $0 \%$ & $90 \%$ to $10 \%$ \\
\hline$\mu_{2}(\mathbf{x})$ & $\pm 5 \%$ to $0 \%$ & $-50 \%$ to $-30 \%$ \\
\hline$\mu_{3}(\mathbf{x})$ & $-30 \%$ to $-2 \%$ & $-80 \%$ to $-50 \%$ \\
\hline$\mu_{4}(\mathbf{x})$ & $\pm 15 \%$ & $-55 \%$ to $-30 \%$ \\
\hline$\mu_{5}(\mathbf{x})$ & $-40 \%$ to $0 \%$ & $-50 \%$ to $-15 \%$ \\
\hline$\mu_{6}(\mathbf{x})$ & $\pm 10 \%$ to $\pm 2 \%$ & $70 \%$ to $5 \%$ \\
\hline
\end{tabular}

For trends, except $\mu_{6}(\mathbf{x})$, for increasing variance and decreasing range values, average normalized error increases, but for the trend function $\mu_{6}(\mathbf{x})$, there is no significant change with variance and range due to the variability of the trend itself.

For the constant trend, $\mu_{1}(\mathbf{x})$, with regular sampling patterns, UnK gives, maximum 5\% larger errors than OrK. As the sampling number increases, UnK gives similar errors with OrK. For random sampling patterns, with constant trend, UnK gives maximum 90\% larger errors than OrK gives. As the sample number increases, UnK gives $10 \%$ larger errors than OrK does.

For the linear trend $\mu_{2}(\mathbf{x})$, with regular sampling patterns relative error $\varepsilon_{r}$ takes as extreme values $\pm 5 \%$, and both OrK and UnK give similar errors; For random sampling patterns, UnK gives, maximum $50 \%$ smaller errors, as the sample number increase, UnK gives maximum 30\% smaller errors. Errors for trend functions $\mu_{1}(\mathbf{x})$ and $\mu_{2}(\mathbf{x})$ is given in Fig. 1.a and Fig. 1.b, respectively, when variance $\sigma^{2}=1.44$, range $a=5$ and for inhibited sampling.

For the second order trend $\mu_{3}(\mathbf{x})$, for regular sampling, the error for the OrK, $\varepsilon_{r}$, decreases from $30 \%$ to $2 \%$ with the increase in sample number; For random sampling these values becomes $80 \%$ to $50 \%$, respectively. As the variance increases both OrK and UnK give similar errors. The errors for the trend $\mu_{3}(\mathbf{x})$, for variances $\sigma^{2}=0.64$ and $\sigma^{2}=2.56$, are given in Fig.2.a and Fig.2.b, respectively, when $a=10$, and for square grid sampling.
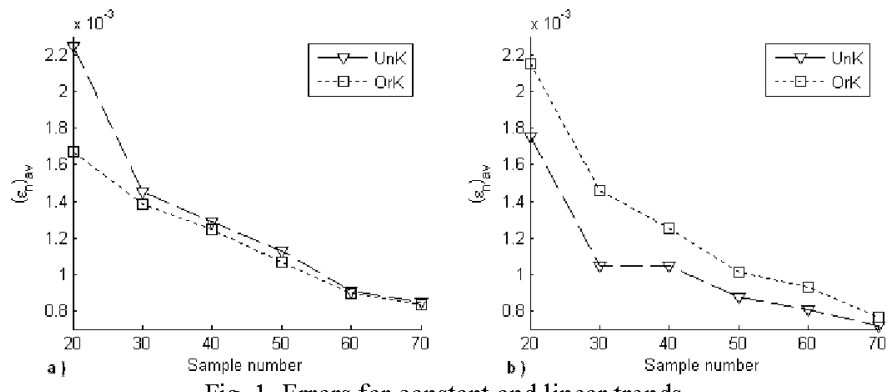

Fig. 1. Errors for constant and linear trends.
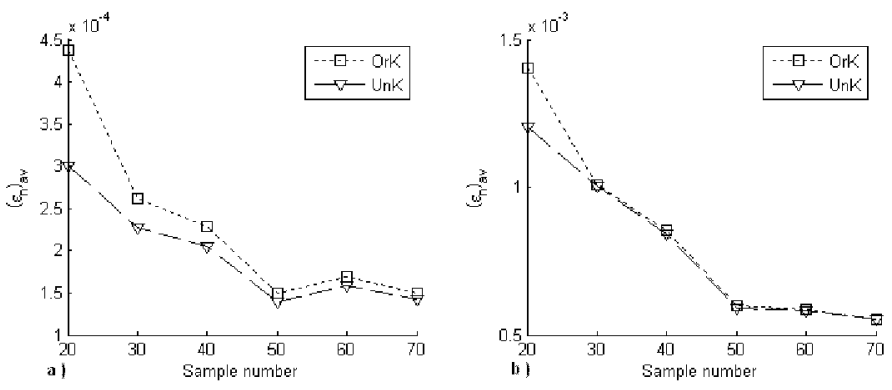

Fig. 2. Errors for $\mu_{3}(\mathbf{x})$, when $\sigma^{2}=0.64$ and $\sigma^{2}=2.56$

When the sampling is random, for uniform and inhibited samplings the UnK errors are similar to the error values when the sampling is regular, but OrK errors are larger than error of OrK with regular sampling. For the cluster process and small variance values, UnK errors are smaller than OrK errors.

When the trend is Gaussian, and the center of the Gaussian surface is on the south of the grid as in $\mu_{4}(\mathbf{x})$, for the regular sampling patterns, OrK errors are similar to UnK errors and OrK gives usually slightly smaller errors; For the random sampling patterns, the error for OrK, $\varepsilon_{\mu}$, decreases from $55 \%$ to $30 \%$. Errors for trends $\mu_{3}(\mathbf{x})$ and $\mu_{4}(\mathbf{x})$ is given in Fig. 3.a and Fig. 3.b, respectively, for uniform sampling, $\sigma^{2}=1.44$, $a=10$.

For the trend $\mu_{5}(\mathbf{x})$, when the center of the grid coincides with the center of the Gaussian curve, errors for the OrK decrease from $40 \%$ to $0 \%$ for the regular sampling and decrease from $50 \%$ to $15 \%$ for the random sampling patterns. As the sample number increases in regular sampling OrK becomes similar to UnK, but for the random sampling UnK still gives 15\% smaller errors. For the more variable trend $\mu_{6}(\mathbf{x})$, for regular sampling patterns, both OrK and UnK give similar errors with maximum $10 \%$ difference; For random sampling patterns, the error for OrK decrease from $70 \%$ to $5 \%$. In Fig. 4.a and Fig. 5.b, average normalized errors versus sample number is given for $\mu_{5}(\mathbf{x})$ and $\mu_{6}(\mathbf{x})$, respectively, when $\sigma^{2}=0.64, a=10$ and for uniform sampling. 

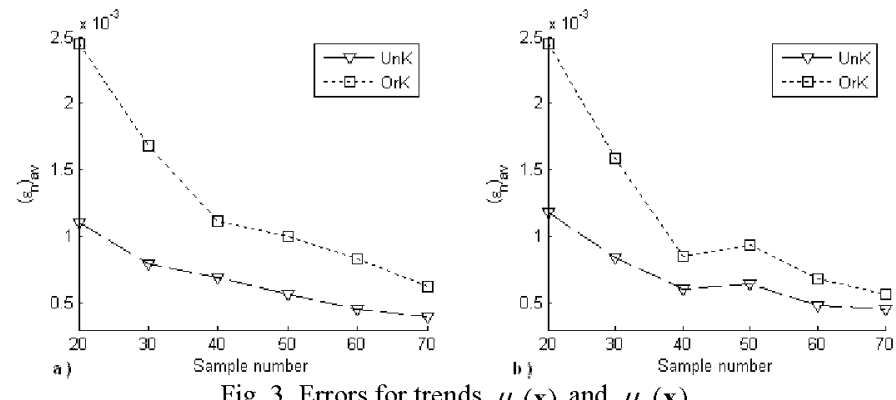

Fig. 3. Errors for trends $\mu_{3}(\mathbf{x})$ and $\mu_{4}(\mathbf{x})$
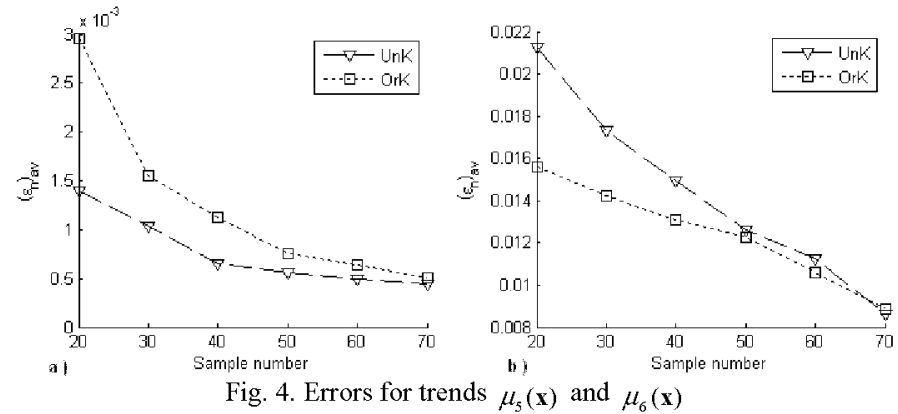

For all variance and range values square, triangular and hexagonal sampling patterns, which are regular, give similar error values smaller than the errors obtained with random sampling patterns. Among the random sampling patterns, inhibited sampling pattern gives the smallest errors, while the clustered sampling pattern gives the largest errors. For the constant trend, UnK errors can approach to OrK errors more rapidly than OrK does for linear or second order trends, as can be seen in Fig. 1., for the linear trend. It can be seen from the Table I., that for regular sampling patterns, errors of both OrK and $\mathrm{UnK}$ are closer to each other relative to random sampling patterns.

\section{CONCLUSION}

In this study, performances of widely used spatial interpolation algorithms in geostatistics, are compared on synthetic TEC surfaces, which represent the various states of the ionosphere. OrK and UnK are run for simulated surfaces for both regular and random sampling patterns using a wide range of sample numbers. The errors between the original surfaces and the interpolated surfaces is measured using averaged normalized differences.

It is observed that, for the constant $\mu_{1}(\mathbf{x})$ and the variable $\mu_{6}(\mathbf{x})$ trends OrK gives smaller error values, while for the other trends, usually, UnK gives smaller errors. When regular sampling patterns are applied, the interpolation errors for both OrK and UnK are similar to each other. When the synthetic surfaces are sampled with random methods, the interpolation error of OrK and UnK differ from each other. The interpolation errors are smaller for constant surfaces when OrK is used. In general interpolation errors decrease with increasing sampling number for both methods. Yet, the errors converge faster for OrK than UnK for the constant trend, and the convergence rate of UnK is generally faster when compared to that of OrK. It is also interesting that, for the Gaussian surface trend $\mu_{4}(\mathbf{x})$, the interpolation errors for $\mathrm{UnK}$ with random sampling is smaller than that of OrK. Yet for regular sampling, OrK results in better reconstruction. As the variability of the surface increase, interpolation with OrK is better.

In the future studies, the space-time variation of the ionosphere will be captured using Kalman-Krige filters.

\section{ACKNOWLEDGMENT}

This study is supported by TUBITTAK EEEAG Grant no: $105 \mathrm{E} 171$.

\section{REFERENCES}

[1] Zimmermann, D., Pavlik, C., Ruggles, A., Armstrong, M.P., "An Experimental Comparison of Ordinary and Universal Kriging and Inverse Distance Weighting," Math. Geo., vol. 31, No.4, 1999.

[2] Yfantis, E.A., Flatman , G.T., Behar, J.V., "Efficiency of Kriging Estimation for Square, Triangular, and Hexagonal Grids", Math. Geo., vol. 19 , no. 3, 1987.

[3] Kyriakidis, P. C., Journel, A., "Geostatistical Space-Time Models", Math. Geo., vol. 31, no. 6, 1999.

[4] Cressie, N. A. C., Statistics for Spatial Data, John Wiley \& Sons, New York, 1993.

[5] Chiles, J. P., Delfiner, P., Geostatistics: modelling spatial uncertainty, John Wiley \& Sons, New York, 1999, 695p.

[6] Wackernagel, H., Multivariate Geostatistics, Springer-Verlag Berlin Heidelberg, New York, 1998, 387p. 